\title{
Insights into chemokine receptors
}

A recent paper published in Science has reported the crystal structure of the chemokine receptor CXCR4, a G protein-coupled receptor (GPCR) that has been implicated in cancer metastasis and HIV infection. This is the first report of a GPCR structure that is activated by a protein ligand, and it shows considerable differences compared with other GPCRs with known crystal structures.

The authors determined the crystal structures of the human CXCR4 in complex with two antagonists: the small molecule antagonist IT1t and the cyclic peptide inhibitor CVX15. As expected, the overall structure of CXCR4 consisted of the canonical bundle of seven transmembrane helices that are typical of all GPCRs. The most notable difference between CXCR4 and other characterized GPCRs (such as rhodopsin, the $\beta_{2}$-adrenergic receptor and the $A_{2 A}$ adenosine receptor) was the different shape and the different location of the ligand binding site. The binding cavity was larger, more open and located closer to the extracellular surface. It was defined by side chains from helices I, II, III and VII but, unlike other known GPCR-ligand complexes, made no contact with helices IV, V and VI.

There were also differences in the arrangement of the transmembrane helices, suggesting that homology modelling (based on previously defined GPCR structures) would be difficult. In addition, CXCR4 lacked several features previously thought to be common to many GPCRs, such as a short alpha helix located at the start of the carboxyl terminus.

CXCR4 is thought to form homo- and hetero-oligomers, and the structures elucidated in this study corroborated this. However, CXCR4 oligomers would form in a considerably different way from previous models of GPCR oligomerization. That is, CXCR4 uses helices V and VII to interact and is stabilized by helices III and IV. Furthermore, the structure of CXCR4 supported the 'two site' model of chemokine signalling, whereby a core sequence of a chemokine ligand docks to the binding domain of the receptor, and the amino terminal domain of the ligand triggers signalling.

Hopefully, knowledge of the structure of CXCR4 will provide new insights into chemokine receptor-ligand interactions, such as the interaction of CXCR4 with its endogenous ligand CXCL12 (which inhibits HIV-1 entry) and with the HIV-1 glycoprotein gp120.

\section{Charlotte Harrison}

\section{ORIGINAL RESEARCH PAPER Structure} of the CXCR4 chemokine GPCR with small molecule and cyclic peptide antagonists. Science 7 Oct 2010 (doi:10.1126/science.1194396)

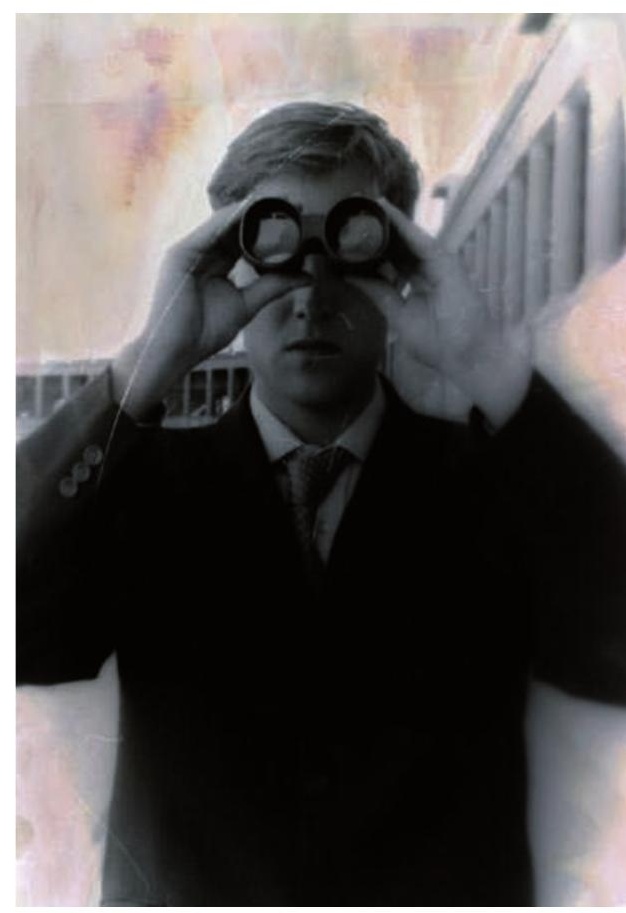

